\title{
Endogenous avidin-binding activity in human lymphoid tissue
}

\author{
DIPONKAR BANERJEE, SABINA PETTIT \\ From the Immunopathology Laboratory, Department of Pathology, University Hospital, London, Ontario, \\ Canada
}

SUMMARY Biotin-avidin systems can be used as an alternative to indirect antibody sandwich methods in the detection of $T$ lymphocyte subsets in cryostat sections of human lymphoid tissue. Appreciable endogenous avidin binding activity (EABA) has been found, however, in human lymph nodes and tonsils. Such EABA can be a source of false positive staining when biotin-avidin detection systems are used to identify cells in cryostat sections. The finding that avidin binding cells may also contain endogenous peroxidase activity and are morphologically similar to histiocytes suggests that such cells may be of histiocytic lineage. EABA is not seen in intrafollicular "tingible body" macrophages, however, and only rarely in medullary sinus histiocytes. Thus further studies are necessary to identify the lineage of avidin binding cells in lymphoid tissues. EABA can be effectively blocked by treatment of cryostat sections with $1 \%$ avidin followed by $0.01 \%$ biotin before specific staining with biotinylated antibodies and avidin-peroxidase or avidin-fluorochrome conjugates.

Biotin-avidin systems can be used to detect antigens in tissue sections. ${ }^{1}$ With the availability of biotinylated monoclonal antibodies against $\mathrm{T}$ lymphocyte subsets, biotin-avidin systems can be used in conjunction with indirect antibody sandwich techniques when two colour fluorescence is desirable to study dual marker expression by cells. ${ }^{2}$ We have found that human tonsils and lymph nodes contain some endogenous avidin binding cells. Such cells can be a potential source of error when measuring any population of cells found in small numbers in human lymphoid tissues. Avidin binding by such cells can be blocked effectively, however, allowing specific staining by biotin-avidin systems.

\section{Material and methods}

Human lymph nodes and tonsils were obtained by surgical excision for diagnostic or therapeutic purposes. Representative samples were frozen in a mixture of isopentane and liquid nitrogen and stored at $-70^{\circ} \mathrm{C}$ until sectioned. Cryostat sections $(6 \mu \mathrm{m})$ were cut and picked up on albuminised slides and dried under a fan for $2 \mathrm{~h}$. The dried sections were fixed for $5 \mathrm{~min}$ in a $1: 1$ chloroform-acetone mixture at room temperature, air dried, and lyophilised.

Accepted for publication 26 October 1983
Lyophilised sections were stored at $-70^{\circ} \mathrm{C}$ until used for immunohistochemistry.

Cryostat sections were rehydrated for $10 \mathrm{~min}$ in phosphate buffered saline (PBS) containing 10\% $A B$ serum. Sections to be stained with avidin horseradish peroxidase (A-HRP) were treated with $3 \%$ hydrogen peroxidase in PBS for $5 \mathrm{~min}$ to block endogenous peroxidase activity. ${ }^{3}$ Unblocked serial sections were used to detect endogenous peroxidase activity. The sections were then incubated with 10 $\mathrm{ml}$ of A-HRP (Vector Laboratories Inc, Burlingame, California) or avidin tetramethyl rhodamine isothiocyanate (A-TRITC) (Becton Dickinson, Sunnyvale, California) for $30 \mathrm{~min}$ at room temperature. The sections were then washed thoroughly. A-HRP incubated slides were incubated with 3.3diaminobenzidine in $0.01 \%$ hydrogen peroxide in PBS for $5 \mathrm{~min}$ and washed, counterstained with haematoxylin, and mounted. A-TRITC incubated slides were mounted in PBS-glycerol after the final wash.

A-TRITC stained sections were viewed with a Zeiss fluorescence microscope by epi-illumination. Fluorescence was recorded on $3 \mathrm{M}$ high speed transparency film (ASA 640). A-HRP preparations were photographed on Kodak Pan X film (ASA 32) using a blue filter.

Endogenous avidin binding activity was blocked 
by the following procedure: sections were pretreated with various concentrations of avidin $(0.01 \%$ to $2 \%)$ for $20 \mathrm{~min}$, washed with PBS for 20 min, and incubated with various concentrations of biotin $(0.001 \%$ to $0.02 \%)$ for $20 \mathrm{~min}^{4}{ }^{4}$ Avidin and biotin were obtained from Sigma Chemical Co, St Louis, Missouri.

\section{Results}

At dilutions of less than 1:300 for A-TRITC and 1:400 for A-HRP, considerable nuclear staining was seen (Fig. 1). At higher dilutions nuclear staining was no longer observed. Persistent, intense cytoplasmic staining, however, was noted in isolated cells in the paracortical regions (Fig. 2). These cells were large mononuclear cells with abundant cytoplasm, resembling histiocytes. Dendritic processes were not seen. Serial sections incubated with 3.3diaminobenzidine alone showed endogenous peroxidase activity in similar cells. Endogenous peroxidase could be completely blocked by $3 \%$ $\mathrm{H}_{2} \mathrm{O}_{2}$. EABA was not found in "tingible-body" macrophages in follicular centres of lymph nodes or tonsils and only rarely within medullary sinuses of lymph nodes.

EABA was effectively blocked by pretreatment with $1 \%$ avidin and $0.01 \%$ biotin (Fig. 3) but not with lower concentrations. Nuclear staining seen at dilutions of less than 1:300 for A-TRITC and 1:400 for A-HRP could be reduced but not completely abolished by pretreatment with avidin and biotin.

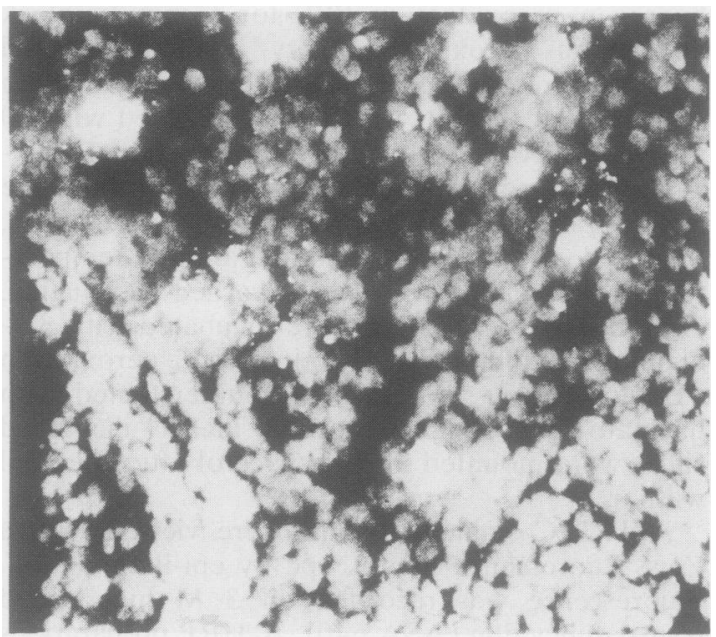

Fig. 1 Nuclear fluorescence in tonsil section incubated with avidin tetramethyl rhodamine isothiocyanate (1:100). $E A B A$ unblocked. Original magnification $\times 400$.

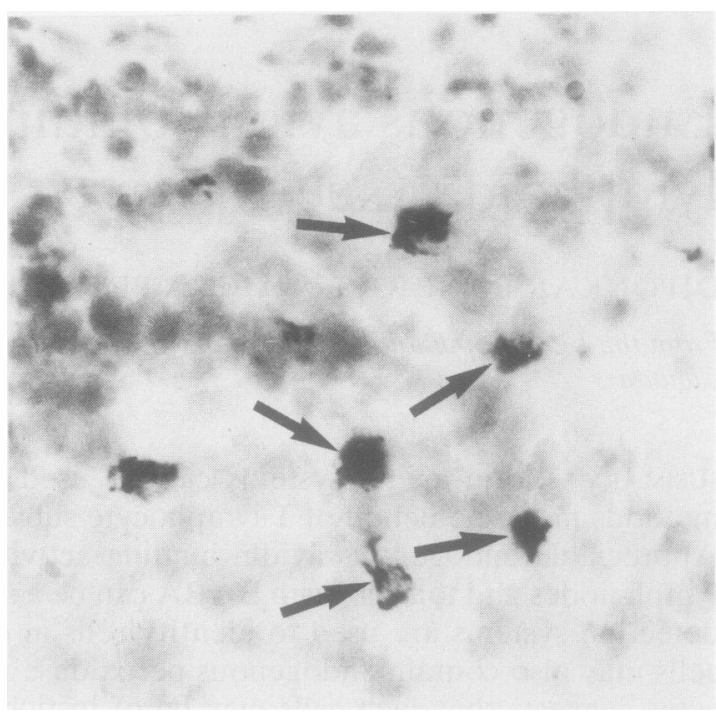

Fig. 2 Persistent cytoplasmic staining (arrows) in tonsil section incubated with avidin-horseradish peroxidase (1:400) without blocking EABA. Haematoxylin counterstain. Endogenous peroxidase blocked. Original magnification $\times 400$.

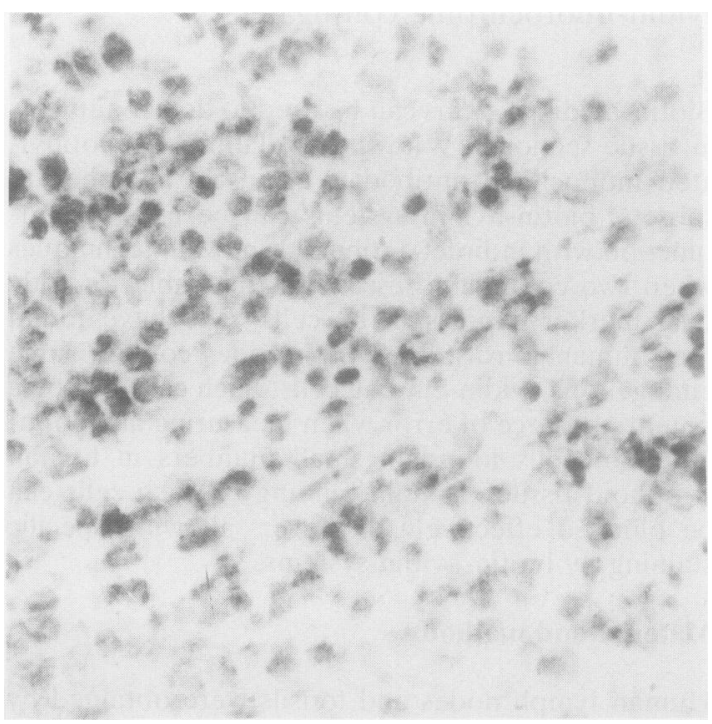

Fig. 3 Complete absence of cytoplasmic staining in tonsil section incubated with avidin-horseradish peroxidase (1:400) after blocking EABA and endogenous peroxidase. Haematoxylin counterstain. Original magnification $\times 250$.

\section{Discussion}

Biotin, a vitamin, is found in various mammalian tissues $^{5}$ and is a potential source of endogenous 
avidin binding activity. Avidin has four binding sites for biotin, ${ }^{6}$ while each biotin molecule can bind only one avidin molecule.' This allows complete saturation of avidin binding sites in tissues with excess free avidin and the subsequent incubation with free biotin then blocks all available biotin binding sites on the avidin molecules. The avidin-biotin immunohistochemical technique can then be used without EABA interfering with the interpretation of the distribution of specific staining.

Avidin conjugates may have a non-specific affinity for chromatin at high concentrations resulting in nuclear staining (Fig. 1) which cannot be effectively blocked by pretreatment with avidin and biotin but can be eliminated by appropriate dilution of avidin conjugates. EABA in this study was defined as cytoplasmic staining which could be inhibited by pretreatment with avidin and biotin.

The distribution of EABA in various tissues has been described by Wood and Warnke, ${ }^{4}$ but these investigators did not find appreciable EABA in lymphoid tissues. This may be because they did not block endogenous peroxidase activity and thus EABA would have been masked if confined to endogenous peroxidase positive cells. Although, in this study the number of cells displaying EABA was small, these could still be a source of error when quantitating any group of cells which are found in small numbers in lymphoid tissues. Effective blocking of EABA in lymphoid tissues requires higher concentrations of avidin and biotin than reported for other tissues by Wood and Warnke. ${ }^{4}$ Since cells with EABA were similar to those with endogenous peroxidase activity it is possible that the EABA positive cells are histiocytes. We have also found numerous avidin binding cells in the lamina propria of intestinal mucosa and in lymph nodes from some patients with Hodgkin's disease (unpublished observations). In both instances the avidin binding cells were large mononuclear cells with abundant cytoplasm, resembling histiocytes. Follicular centre histiocytes ("tingible-body" macrophages) and most medullary sinus histiocytes do not express EABA, however, and the paracortical cells with EABA may therefore represent some other cell population. Further studies are necessary to identify the lineage of EABA positive paracortical cells of human lymphoid tissue.

We are grateful to Miss Colleen Schwartzel for typing this manuscript.

\section{References}

' Guesdon JL, Ternynck T, Avrameas S. The use of avidin-biotin interaction in immunoenzymatic techniques. $J$ Histochem Cytochem 1979;27:1131-9.

${ }^{2}$ Banerjee D, Thibert RF. Natural killer-like cells found in B cell compartments of human lymphoid tissues. Nature 1983; 304:270-2.

${ }^{3}$ Burns J. Background staining and sensitivity of the unlabelled antibody-enzyme (PAP) method. Comparison with peroxidase labelled antibody sandwich method using formalin fixed paraffin embedded material. Histochemistry 1975;43:291-4.

${ }^{4}$ Wood GS, Warnke R. Suppression of endogenous avidin-binding activity in tissues and its relevance to biotin-avidin detection systems. J Histochem Cytochem 1981;29:1196-204.

${ }^{5}$ Dakshinamurti K, Mistry SP. Tissue and intracellular distribution of biotin- $\mathrm{C}^{14} \mathrm{OOH}$ in rats and chicks. $J$ Biol Chem 1963;238:294-6.

- Green NM. Avidin. Adv Protein Chem 1975;29:85-133.

' Moss J, Lane MD. The biotin-dependent enzymes. Adv Enzymol 1971;35:321-442.

Requests for reprints to: Dr D Banerjee, Department of Pathology, 4026 Health Sciences Centre, University of Western Ontario, London, Ontario N6A 5C1, Canada. 